\title{
Stochastic Integration with Respect to Cylindrical Lévy Processes by $p$-Summing Operators
}

\author{
Tomasz Kosmala $^{1} \cdot$ Markus Riedle $^{1}(\mathbb{C}$ \\ Received: 20 September 2019 / Published online: 4 January 2020 \\ (c) The Author(s) 2020
}

\begin{abstract}
We introduce a stochastic integral with respect to cylindrical Lévy processes with finite $p$-th weak moment for $p \in[1,2]$. The space of integrands consists of $p$-summing operators between Banach spaces of martingale type $p$. We apply the developed integration theory to establish the existence of a solution for a stochastic evolution equation driven by a cylindrical Lévy process.
\end{abstract}

Keywords Cylindrical Lévy processes - Stochastic integration in Banach spaces · Stochastic partial differential equations $\cdot p$-Summing operators

Mathematics Subject Classification (2010) 47B10 · 60G51 · 46T12 · 60H15

\section{Introduction}

Cylindrical Lévy processes are a natural generalisation of cylindrical Brownian motions to the non-Gaussian setting, and they can serve as a model of random perturbation of partial differential equations or other dynamical systems. As a generalised random process, cylindrical Lévy processes do not attain values in the underlying space, and they do not enjoy a Lévy-Itô decomposition in general. Since conventional approaches to stochastic integration rely on either stopping time arguments or a semimartingale decomposition in the one or other form, a completely novel method for stochastic integration has been introduced in the work [12] by one of us with Jakubowski. This method is purely based on tightness arguments, since typical estimates of the expectation are not available without utilising stopping time arguments or a semimartingale decomposition. As a consequence, although this method guarantees the existence of the stochastic integral for a large class of random integrands, it does

$\otimes \quad$ Markus Riedle
markus.riedle@kcl.ac.uk
Tomasz Kosmala
tomasz.kosmala@kcl.ac.uk
$1 \quad$ Department of Mathematics, King's College London, London WC2R 2LS, UK 
not provide any control of the integrals. Since many applications, such as modelling dynamical systems or control problems, require upper estimates of the stochastic integrals, this method seems to be difficult to use for such applications.

In order to provide a control of the stochastic integral, we develop a theory of stochastic integration for random integrands with respect to cylindrical Lévy processes with finite $p$-th weak moments for $p \in[1,2]$ in this work. Our approach enables us to develop the theory on a large class of general Banach spaces. We apply the obtained estimates to establish the existence of an abstract partial differential equations driven by a cylindrical Lévy process with finite $p$-th weak moments.

Stochastic integration with respect to a cylindrical Wiener process is well developed in Hilbert spaces and various classes of Banach spaces. Typical Banach spaces which permit a development of stochastic integration are martingale type 2 Banach spaces, see, for example, Dettweiler [7,8], or UMD spaces, see, for example, van Neerven, Veraar and Weis [32]. Veraar and Yaroslavtsev [33] extend the approach for UMD spaces in [32] to continuous cylindrical local martingales by utilising the DambisDubins-Schwarz Theorem. Stochastic integration in Hilbert spaces with respect to genuine Lévy processes is, for example, presented by Peszat and Zabczyk in [18], and with respect to cylindrical Lévy processes, the theory is developed in [12]. Stochastic integration with respect to a Poisson random measure in Banach spaces is developed, for example, by Mandrekar and Rüdiger in [17] and by Brzeźniak, Zhu and Hausenblas [34].

In this work, we are faced with the similar problem as in [12]. Conventional approaches to stochastic integration utilise either stopping times or the Lévy-Itô decomposition to show continuity of the integral operator separately: firstly with respect to the martingale part with finite second moments and secondly with respect to the bounded variation part. However, since these approaches are excluded for cylindrical Lévy processes, we show continuity of the integral operator "in one piece", i.e. without applying the semimartingale decomposition of the integrator. For this purpose, we utilise a generalised form of Pietsch's factorisation theorem, originating from the work of Schwartz [29].

More specifically, the space of admissible integrands is predictable stochastic process with values in the space of $p$-summing operators and with integrable $p$-summing norm for $p \in[1,2]$ in this work. Due to results by Kwapień and Schwartz, for $p>1$ the space of $p$-summing operators coincides with the space of $p$-radonifying operators, which are exactly the operators which map each cylindrical random variable with finite $p$-th weak moments to a genuine random variable. In this way, stochastic processes with values in the space of $p$-summing operators are the natural class of integrands, as they map the cylindrical increments of the integrator to the genuine random variables. Furthermore, the class of $p$-summing operators coincides with the class of Hilbert-Schmidt operators in Hilbert spaces, and as such, the aforementioned space of admissible integrands is a natural generalisation of the integration theory in Hilbert spaces with respect to genuine Lévy process in, for example, [18]. In typical applications such as the heat equation, the $p$-summing norm of the operators appearing in the equation can be explicitly estimated, see [3].

In Sect. 2, we recall the concepts of cylindrical measures and cylindrical Lévy processes. In Sect. 3, we present the generalised Pietsch's factorisation theorem due 
to Schwartz and derive a result on the strong convergence of $p$-summing operators, which is needed in the proof of the stochastic continuity of the stochastic convolution. Section 4 is devoted to the construction of the stochastic integral. This is done in two steps as in the article [22] by the second author. Firstly, we radonify the increments of the cylindrical Lévy process by random $p$-summing operators. Secondly, we define the integral for simple integrands and extend it by continuity to the general ones. We also present some examples of the processes covered by our theory. In Sect. 5, we apply our results to establish existence and uniqueness solution to the evolution equation driven by a cylindrical Lévy noise with finite $p$-th weak moments for $p \in[1,2]$.

\section{Preliminaries}

Let $E$ and $F$ be Banach spaces with separable duals $E^{*}$ and $F^{*}$. The operator norm of an operator $u: E \rightarrow F$ is denoted with $\|u\|_{\mathcal{L}(E, F)}$ or simply $\|u\|$. We write $B_{E}$ for the closed unit ball in $E$. The Borel $\sigma$-field is denoted with $\mathcal{B}(E)$.

Fix a probability space $(\Omega, \mathcal{F}, P)$ with a filtration $\left(\mathcal{F}_{t}\right)$. We denote the space of equivalence classes of real-valued random variables equipped with the topology of convergence in probability by $L^{0}(\Omega, \mathcal{F}, P ; \mathbb{R})$. The Bochner space of equivalence classes of $E$-valued random variables with finite $p$-th moment is denoted with $L^{p}(\Omega, \mathcal{F}, P ; E)$. In case the codomain is not separable, we take only separably valued random variables.

Cylindrical sets are sets of the form

$$
C\left(x_{1}^{*}, \ldots, x_{n}^{*} ; B\right)=\left\{x \in E:\left(x_{1}^{*}(x), \ldots x_{n}^{*}(x)\right) \in B\right\}
$$

for $x_{1}^{*}, \ldots, x_{n}^{*} \in E^{*}$ and $B \in \mathcal{B}\left(\mathbb{R}^{n}\right)$. For $\Gamma \subseteq E^{*}$, we denote with $\mathcal{Z}(E, \Gamma)$ the collection of all cylindrical sets with $x_{1}^{*}, \ldots, x_{n}^{*} \in \Gamma, B \in \mathcal{B}\left(\mathbb{R}^{n}\right)$ and $n \in \mathbb{N}$. If $\Gamma=E^{*}$, we write $\mathcal{Z}(E)$ to denote the collection of all cylindrical subsets of $E$. Note that $\mathcal{Z}(E)$ is an algebra and that if $\Gamma$ is finite, then $\mathcal{Z}(E, \Gamma)$ is a $\sigma$-algebra. A function $\mu: \mathcal{Z}(E) \rightarrow[0, \infty]$ is called a cylindrical measure if its restriction to $\mathcal{Z}(E, \Gamma)$ is a measure for every finite subset $\Gamma \subseteq E^{*}$. If $\mu(E)=1$, we call it a cylindrical probability measure. A cylindrical random variable is a linear and continuous mapping:

$$
Y: E^{*} \rightarrow L^{0}(\Omega, \mathcal{F}, P ; \mathbb{R})
$$

The cylindrical distribution of a cylindrical random variable $Y$ is defined by

$$
\mu\left(C\left(x_{1}^{*}, \ldots, x_{n}^{*} ; B\right)\right)=P\left(\left(Y x_{1}^{*}, \ldots, Y x_{n}^{*}\right) \in B\right),
$$

which defines a cylindrical probability measure on $\mathcal{Z}(E)$. The characteristic function of a cylindrical random variable (resp. cylindrical probability measure) is given by

$$
\phi_{Y}\left(x^{*}\right)=\mathbb{E}\left[e^{i Y x^{*}}\right], \quad\left(\operatorname{resp} . \phi_{\mu}\left(x^{*}\right)=\int_{E} e^{i x^{*}(x)} \mu(\mathrm{d} x)\right),
$$


for $x^{*} \in E^{*}$. We say that a cylindrical random variable $Y$ (resp. cylindrical measure $\mu$ ) is of weak order $p$ or has finite $p$-th weak moments if $\mathbb{E}\left[\left|Y x^{*}\right|^{p}\right]<\infty$ for every $x^{*} \in E^{*}$ (resp. $\left.\int_{E}\left|x^{*}(x)\right|^{p} \mu(\mathrm{d} x)<\infty\right)$. We say that $Y$ is induced by an $E$-valued random variable $X: \Omega \rightarrow E$ if

$$
Y x^{*}=x^{*}(X) \quad \text { for all } x^{*} \in E^{*} .
$$

A family of cylindrical random variables $(L(t): t \geq 0)$ is called a cylindrical Lévy process if, for every $x_{1}^{*}, \ldots, x_{n}^{*} \in E^{*}$ and $n \in \mathbb{N}$, we have that

$$
\left(\left(L(t) x_{1}^{*}, \ldots, L(t) x_{n}^{*}\right): t \geq 0\right)
$$

is a Lévy process in $\mathbb{R}^{n}$ with respect to the filtration $\left(\mathcal{F}_{t}\right)$. We say that $L$ is weakly $p$-integrable if $\mathbb{E}\left[\left|L(1) x^{*}\right|^{p}\right]<\infty$ for every $x^{*} \in E^{*}$. The characteristic function of $L$ (1) can be written in the form:

$$
\begin{aligned}
\phi_{L(1)}\left(x^{*}\right)= & \exp \left(i p\left(x^{*}\right)-\frac{1}{2} q\left(x^{*}\right)\right. \\
& \left.+\int_{E}\left(e^{i x^{*}(x)}-1-i x^{*}(x) \mathbb{1}_{B_{\mathbb{R}}}\left(x^{*}(x)\right)\right) v(\mathrm{~d} x)\right),
\end{aligned}
$$

where $p: E^{*} \rightarrow \mathbb{R}$ is a continuous function with $p(0)=0, q: E^{*} \rightarrow \mathbb{R}$ is a quadratic form, and $v$ is a finitely additive set function on cylindrical sets of the form $C\left(x_{1}^{*}, \cdots, x_{n}^{*} ; B\right)$ for $x_{1}^{*}, \ldots, x_{n}^{*} \in E^{*}$ and $B \in \mathcal{B}\left(\mathbb{R}^{n} \backslash\{0\}\right)$, such that for every $x^{*} \in E^{*}$, it satisfies

$$
\int_{\mathbb{R} \backslash\{0\}}\left(|\beta|^{2} \wedge 1\right)\left(\nu \circ\left(x^{*}\right)^{-1}\right)(\mathrm{d} x)<\infty .
$$

Cylindrical Lévy processes are introduced in [1], and further details on the characteristic function can be found in [21].

An operator $u: E \rightarrow F$ is called $p$-summing if there exists a constant $c$ such that

$$
\left(\sum_{k=1}^{n}\left\|u\left(x_{k}\right)\right\|^{p}\right)^{1 / p} \leq c \sup \left\{\left(\sum_{k=1}^{n}\left|x^{*}\left(x_{k}\right)\right|^{p}\right)^{1 / p}: x^{*} \in B_{E^{*}}\right\}
$$

for all $x_{1}, \ldots, x_{n} \in E$ and $n \in \mathbb{N}$; see [9]. We denote with $\pi_{p}(u)$ its $p$-summing norm, which is the smallest possible constant $c$ in (1). The space of $p$-summing operators is denoted with $\Pi_{p}(E, F)$. If $E$ and $F$ are Hilbert spaces, this space coincides with the space of Hilbert-Schmidt operators denoted by $L_{\mathrm{HS}}(E, F)$ with the norm $\|\cdot\|_{L_{\mathrm{HS}}(E, F)}$; see [9, Th. 4.10 and Cor. 4.13]. Moreover, the $p$-summing norms and the HilbertSchmidt norm in $L_{\mathrm{HS}}(E, F)$ are equivalent.

A Banach space $E$ is of martingale type $p \in[1,2]$ if there exists a constant $C_{p}$ such that for all finite $E$-valued martingales $\left(M_{k}\right)_{k=1}^{n}$ the following inequality is satisfied:

$$
\sup _{k=1, \ldots, n} \mathbb{E}\left[\left\|M_{k}\right\|^{p}\right] \leq C_{p} \sum_{k=1}^{n} \mathbb{E}\left[\left\|M_{k}-M_{k-1}\right\|^{p}\right],
$$


where we use the convention that $M_{0}=0$; see [11].

We use the notation $u(\mu)$ for the push-forward cylindrical measure $\mu \circ u^{-1}$ for a continuous linear function $u: E \rightarrow F$ and a cylindrical measure $\mu$. An operator $u: E \rightarrow F$ is called $p$-radonifying for some $p \geq 0$ if for every cylindrical measure $\mu$ on $E$ of weak order $p$, the measure $u(\mu)$ extends to a Radon measure on $F$ with finite $p$-th strong moment. Equivalently, the mapping $u$ is $p$-radonifying if for every cylindrical random variable $Y$ on $E^{*}$ with finite weak $p$-th moment, the cylindrical random variable $Y \circ u^{*}$ is induced by an $F$-valued random variable with finite $p$-th strong moment; see [31, Prop. VI.5.2].

A Banach space $E$ has the approximation property if for every compact set $K \subseteq$ $E$ and for every $\epsilon>0$ there exists a finite rank operator $u: E \rightarrow E$ such that $\|u(x)-x\| \leq \epsilon$ for $x \in K$.

A Banach space $E$ has the Radon-Nikodym property if for any probability space $(\Omega, \mathcal{F}, P)$ and vector-valued measure $\mu: \mathcal{F} \rightarrow E$, which is absolutely continuous with respect to $P$, there exists $f \in L^{1}(\Omega, \mathcal{F}, P ; E)$ such that

$$
\mu(A)=\int_{A} f(\omega) P(\mathrm{~d} \omega) \quad \text { for all } A \in \mathcal{F} .
$$

It is well known that every reflexive Banach space has the Radon-Nikodym property; see [31, Cor. 2, p. 219]. It follows from [31, Th. VI.5.4 and Th. VI.5.5] that if either $p>1$ or $p=1$ and $F$ has the Radon-Nikodym property, then the classes of $p$ radonifying and $p$-summing operators between $E$ and $F$ coincide.

\section{Some Results on $p$-Summing Operators}

Our approach to stochastic integration with respect to a cylindrical Lévy process is based on a generalisation of Pietsch's factorisation theorem, which is due to Schwartz; see [30, pp. 23-28] and [28]. For a measure $\mu$ on $\mathcal{B}(E)$ and $p \in[1,2]$, we define

$$
\|\mu\|_{p}:=\left(\int_{E}\|x\|^{p} \mu(d x)\right)^{1 / p},
$$

and say that $\mu$ is of order $p$ if $\|\mu\|_{p}<\infty$. For a cylindrical measure $\mu$ on $\mathcal{Z}(E)$, we define

$$
\|\mu\|_{p}^{*}=\sup _{x^{*} \in B_{E^{*}}}\left\|x^{*}(\mu)\right\|_{p}
$$

and we say that $\mu$ is of weak order $p$ if $\|\mu\|_{p}^{*}<\infty$.

Theorem 1 For $p \in[1,2]$, assume either that $p>1$ or that $F$ has the RadonNikodym property if $p=1$. Each cylindrical probability measure $\mu$ on $\mathcal{Z}(E)$ and $p$-summing map $u: E \rightarrow F$ satisfy

$$
\|u(\mu)\|_{p} \leq \pi_{p}(u)\|\mu\|_{p}^{*} .
$$


Proof See [30] or [28,29].

Remark 2 Pietsch's factorisation theorem states that if $u: E \rightarrow F$ is a $p$-summing map, then there exists a regular probability measure $\rho$ on $B_{E^{*}}$ such that

$$
\|u x\| \leq \pi_{p}(u)\left(\int_{B_{E^{*}}}\left|x^{*}(x)\right|^{p} \rho(\mathrm{d} x)\right)^{1 / p} \quad \text { for all } x \in E .
$$

If $X$ is a genuine random variable $X: \Omega \rightarrow E$ with probability distribution $\mu$ on $\mathcal{B}(E)$, Pietsch's factorisation theorem immediately implies

$$
\|u(\mu)\|_{p}^{p}=\mathbb{E}\left[\|u X\|^{p}\right] \leq\left(\pi_{p}(u)\right)^{p} \mathbb{E}\left[\int_{B_{E^{*}}}\left|x^{*}(X)\right|^{p} \rho(\mathrm{d} x)\right] \leq\left(\pi_{p}(u)\right)^{p}\|\mu\|_{p}^{p} .
$$

For this reason, we refer to Theorem 1 as generalised Pietsch's factorisation theorem.

For establishing the stochastic continuity of the stochastic convolution in Sect. 5, we need a result on the convergence of $p$-summing operators between Banach spaces. In the case of Hilbert spaces, this convergence result can easily be seen: suppose that $U$ and $H$ are separable Hilbert spaces and let $\psi: U \rightarrow H$ be a Hilbert-Schmidt operator. If $\left(\phi_{n}\right)$ is a sequence of operators $\phi_{n}: H \rightarrow H$ converging strongly to 0 as $n \rightarrow \infty$, then the composition $\phi_{n} \psi$ converges to 0 in the Hilbert-Schmidt norm. Indeed, take $\left(e_{n}\right)$ an orthonormal basis of $U$ and calculate

$$
\left\|\phi_{n} \psi\right\|_{L_{\mathrm{HS}}(U, H)}^{2}=\sum_{k=1}^{\infty}\left\|\phi_{n} \psi e_{k}\right\|^{2}
$$

Every term in the above sum converges to 0 as $n \rightarrow \infty$ due to the strong convergence of $\phi_{n}$. By Lebesgue's dominated convergence theorem, we obtain $\left\|\phi_{n} \psi\right\|_{L_{\mathrm{HS}}(U, H)}^{2} \rightarrow 0$. The following result extends this conclusion in Hilbert spaces to the Banach space setting by approximating $p$-summing operators with finite rank operators.

Theorem 3 Suppose that $E$ is a reflexive Banach space or a Banach space with separable dual and that $E^{* *}$ has the approximation property. If $\psi: E \rightarrow F$ is a p-summing operator and $\left(\phi_{n}\right)$ is a sequence of operators $\phi_{n}: F \rightarrow F$ converging strongly to 0 , then we have

$$
\pi_{p}\left(\phi_{n} \psi\right) \rightarrow 0
$$

Proof We first prove the assertion for finite rank operators $\psi: E \rightarrow F$, in which case we can assume that $\psi=\sum_{k=1}^{N} x_{k}^{*} \otimes y_{k}$ for some $x_{k}^{*} \in E^{*}$ and $y_{k} \in F$. Then, $\phi_{n} \psi=\sum_{k=1}^{N} x_{k}^{*} \otimes\left(\phi_{n} y_{k}\right)$ and since $\pi_{p}\left(x^{*} \otimes y\right)=\left\|x^{*}\right\|\|y\|$ by a simple argument (see [9, p. 37]), we estimate

$$
\pi_{p}\left(\phi_{n} \psi\right) \leq \sum_{k=1}^{N} \pi_{p}\left(x_{k}^{*} \otimes\left(\phi_{n} y_{k}\right)\right)=\sum_{k=1}^{N}\left\|x_{k}^{*}\right\|\left\|\phi_{n} y_{k}\right\| \rightarrow 0
$$


because $\left\|\phi_{n} y_{k}\right\| \rightarrow 0$ for every $k \in\{1, \ldots, N\}$.

Consider now the case of a general $p$-summing operator $\psi$. Under the assumptions on $E$ and $F$, by Corollary 1 in [26], the finite rank operators are dense in the space of $p$-summing operators. That is, there exists a sequence of finite rank operators $\left(\psi_{k}\right)$ such that $\pi_{p}\left(\psi_{k}-\psi\right) \rightarrow 0$ as $k \rightarrow \infty$. It follows that

$$
\pi_{p}\left(\phi_{n} \psi\right) \leq \pi_{p}\left(\phi_{n} \psi_{k}\right)+\pi_{p}\left(\phi_{n}\left(\psi-\psi_{k}\right)\right) \quad \text { for all } k, n \in \mathbb{N} \text {. }
$$

Fix $\epsilon>0$, and let $c:=\sup \left\{\left\|\phi_{n}\right\|: n \in \mathbb{N}\right\}$. Choose $k \in \mathbb{N}$ such that $\pi_{p}\left(\psi-\psi_{k}\right) \leq \frac{\epsilon}{2 c}$. Since $\psi_{k}$ is a finite rank operator, the argument above guarantees that there exists $n_{0} \in \mathbb{N}$ such that for all $n \geq n_{0}$, we have $\pi_{p}\left(\phi_{n} \psi_{k}\right) \leq \frac{\epsilon}{2}$. Inequality (5) implies for all $n \geq n_{0}$ that $\pi_{p}\left(\phi_{n} \psi\right) \leq \frac{\epsilon}{2}+\frac{\epsilon}{2}$, which completes the proof.

Remark 4 The proof of Theorem 3 relies on the density of finite rank operators in the space of $p$-summing operators. This holds under more general assumption than assumed in Theorem 3; see [26, pp. 384 and 388].

However, the result of Theorem 3 does not hold in the case of arbitrary Banach spaces as the following example shows. Choose $E=\ell^{1}(\mathbb{R})$ and $F=\ell^{2}(\mathbb{R})$, and equip both spaces with the canonical basis $\left(e_{n}\right)$, where $e_{n}=(0, \ldots, 0,1,0, \ldots)$. We take $\psi=\mathrm{Id}: E \rightarrow F$, which is 1-radonifying by Grothendieck's theorem; see [9, p. 38-39]. Furthermore, we define $\phi_{n}=e_{n} \otimes e_{n}$, i.e. $\phi_{n}(x)=x(n) e_{n}=(0, \ldots, 0, x(n), 0, \ldots)$ for a sequence $x=(x(n)) \in \ell^{2}(\mathbb{R})$. Then, $\phi_{n}$ converges to 0 strongly as $n \rightarrow \infty$, but since $\phi_{n} \psi$ is finite rank, we have $\pi_{1}\left(\phi_{n} \psi\right)=\left\|e_{n}\right\|\left\|e_{n}\right\|=1$ for all $n \in \mathbb{N}$. This counterexample shows that the assumptions on the space $E$ in Theorem 3 cannot be dropped.

\section{Radonification of Increments and Stochastic Integral}

In this section, we fix $p \in[1,2]$ and assume that the cylindrical Lévy process $L$ has finite $p$-th weak moments and assume either that $p>1$ or that $F$ has the RadonNikodym property if $p=1$.

Fix $0 \leq s<t \leq T$. An $\mathcal{F}_{s}$-measurable random variable $\Psi: \Omega \rightarrow \Pi_{p}(E, F)$ is called simple if it is of the form:

$$
\Psi=\sum_{k=1}^{m} \mathbb{1}_{A_{k}} \psi_{k},
$$

for some disjoint sets $A_{1}, \ldots, A_{m} \in \mathcal{F}_{s}$ and $\psi_{1}, \ldots, \psi_{m} \in \Pi_{p}(E, F)$. The space of simple, $\mathcal{F}_{S}$-measurable random variables is denoted with $S:=S\left(\Omega, \mathcal{F}_{s} ; \Pi_{p}(E, F)\right)$, and it is equipped with the norm $\|\Psi\|_{S, p}:=\left(\mathbb{E}\left[\pi_{p}(\Psi)^{p}\right]\right)^{1 / p}$.

Since for $p>1$ or for $p=1$ with $F$ having the Radon-Nikodym property, each $p$-summing operator $\psi_{k}: E \rightarrow F$ is $p$-radonifying, it follows that the cylindrical random variable $(L(t)-L(s)) \psi_{k}^{*}$ is induced by a classical, $F$-valued random variable $X_{\psi_{k}}: \Omega \rightarrow F:$

$$
(L(t)-L(s)) \psi_{k}^{*}\left(x^{*}\right)=x^{*}\left(X_{\psi_{k}}\right) \quad \text { for all } x^{*} \in F^{*} .
$$


This enables us to define the operator

$$
J_{s, t}: S\left(\Omega, \mathcal{F}_{s} ; \Pi_{p}(E, F)\right) \rightarrow L^{p}(\Omega, \mathcal{F}, P ; F), \quad J_{s, t}(\Psi):=\sum_{k=1}^{m} \mathbb{1}_{A_{k}} X_{\psi_{k}}
$$

The following result allows us to extend the operator $J_{s, t}$ to $L^{p}\left(\Omega, \mathcal{F}_{s}, P ; \Pi_{p}(E, F)\right)$.

Lemma 5 (Radonification of the increments) For fixed $0 \leq s<t \leq T$, the operator $J_{s, t}$ defined in (7) is continuous and satisfies

$$
\left\|J_{s, t}\right\|_{\mathcal{L}\left(S, L^{p}\right)} \leq\|L(t-s)\|_{\mathcal{L}\left(E^{*}, L^{p}(\Omega ; \mathbb{R})\right)},
$$

and thus, $J_{s, t}$ can be extended to $J_{s, t}: L^{p}\left(\Omega, \mathcal{F}_{s}, P ; \Pi_{p}(E, F)\right) \rightarrow L^{p}\left(\Omega, \mathcal{F}_{t}, P ; F\right)$.

Proof Let $\Psi$ be of the form (6). Since the sets $A_{k}$ are disjoint, it follows that

$$
\mathbb{E}\left[\left\|J_{S, t}(\Psi)\right\|^{p}\right]=\mathbb{E}\left[\left\|\sum_{k=1}^{m} \mathbb{1}_{A_{k}} X_{\psi_{k}}\right\|^{p}\right]=\mathbb{E}\left[\sum_{k=1}^{m} \mathbb{1}_{A_{k}}\left\|X_{\psi_{k}}\right\|^{p}\right] .
$$

Using the fact that each $A_{k}$ is $\mathcal{F}_{s}$-measurable and that $X_{\psi_{k}}$ is independent of $\mathcal{F}_{s}$, we can calculate further

$$
\mathbb{E}\left[\left\|J_{s, t}(\Psi)\right\|^{p}\right]=\sum_{k=1}^{m} \mathbb{E}\left[\mathbb{E}\left[\mathbb{1}_{A_{k}}\left\|X_{\psi_{k}}\right\|^{p} \mid \mathcal{F}_{s}\right]\right]=\sum_{k=1}^{m} P\left(A_{k}\right) \mathbb{E}\left[\left\|X_{\psi_{k}}\right\|^{p}\right] .
$$

In order to estimate $\mathbb{E}\left[\left\|X_{\psi_{k}}\right\|^{p}\right]$, we apply Theorem 1 to obtain that

$$
\left(\mathbb{E}\left[\left\|X_{\psi_{k}}\right\|^{p}\right]\right)^{1 / p} \leq \pi_{p}\left(\psi_{k}\right)\|L(t)-L(s)\|_{p}^{*} .
$$

Since stationary increments of the real-valued Lévy processes yield

$$
\left(\mathbb{E}\left[\left|(L(t)-L(s)) x^{*}\right|^{p}\right]\right)^{1 / p}=\left(\mathbb{E}\left[\left|L(t-s) x^{*}\right|^{p}\right]\right)^{1 / p} \quad \text { for all } x^{*} \in E^{*},
$$

it follows that

$$
\|L(t)-L(s)\|_{p}^{*}=\sup _{x^{*} \in B_{E^{*}}}\left(\mathbb{E}\left[\left|L(t-s) x^{*}\right|^{p}\right]\right)^{1 / p}=\|L(t-s)\|_{\mathcal{L}\left(E^{*}, L^{p}(\Omega ; \mathbb{R})\right)} .
$$

Note that by the closed graph theorem and the continuity of $L(t-s): E^{*} \rightarrow$ $L^{0}(\Omega, \mathcal{F}, P ; \mathbb{R})$, the mapping $L(t-s): E^{*} \rightarrow L^{p}(\Omega, \mathcal{F}, P ; \mathbb{R})$ is continuous. This 
shows that the last expression in (11) is finite. Applying estimates (10) and (11) to (9) results in

$$
\begin{aligned}
\left(\mathbb{E}\left[\left\|J_{s, t}(\Psi)\right\|^{p}\right]\right)^{1 / p} & \leq\left(\sum_{k=1}^{m} P\left(A_{k}\right) \pi_{p}\left(\psi_{k}\right)^{p}\|L(t-s)\|_{\mathcal{L}\left(E^{*}, L^{p}(\Omega ; \mathbb{R})\right)}^{p}\right)^{1 / p} \\
& =\|L(t-s)\|_{\mathcal{L}\left(E^{*}, L^{p}(\Omega ; \mathbb{R})\right)}\left(\mathbb{E}\left[\pi_{p}(\Psi)^{p}\right]\right)^{1 / p}
\end{aligned}
$$

which proves (8).

For defining the stochastic integral below, let $\Lambda\left(\Pi_{p}(E, F)\right)$ denote the space of predictable processes $\Psi:[0, T] \times \Omega \rightarrow \Pi_{p}(E, F)$ such that

$$
\|\Psi\|_{\Lambda}:=\left(\mathbb{E}\left[\int_{0}^{T} \pi_{p}(\Psi(s))^{p} \mathrm{~d} s\right]\right)^{1 / p}<\infty
$$

that is, $\Lambda\left(\Pi_{p}(E, F)\right)=L^{p}\left([0, T] \times \Omega, \mathcal{P}, \mathrm{d} t \otimes P ; \Pi_{p}(E, F)\right)$, where $\mathcal{P}$ denotes the predictable $\sigma$-algebra on $[0, T] \times \Omega$. A simple stochastic process is of the form:

$$
\Psi(t)=\Psi_{0} \mathbb{1}_{\{0\}}(t)+\sum_{k=1}^{N-1} \Psi_{k} \mathbb{1}_{\left(t_{k}, t_{k+1}\right]}(t),
$$

where $0=t_{1}<\cdots<t_{N}=T$, and each $\Psi_{k}$ is an $\mathcal{F}_{t_{k}}$-measurable, $\Pi_{p}(E, F)$-valued random variable with $E\left[\pi_{p}\left(\Psi_{k}\right)^{p}\right]<\infty$. We denote with $\Lambda_{0}^{S}\left(\Pi_{p}(E, F)\right)$ the space of simple processes of the form (13) where each $\Psi_{k}$ is a simple random variable of the form (6), i.e. taking only a finite number of values.

Since for stochastic processes in $\Lambda_{0}^{S}\left(\Pi_{p}(E, F)\right)$, the radonification of the increments is defined by the operator $J_{s, t}$, we can define the integral operator by

$$
I: \Lambda_{0}^{S}\left(\Pi_{p}(E, F)\right) \rightarrow L^{p}\left(\Omega, \mathcal{F}_{T}, P ; F\right), \quad I(\Psi):=\sum_{k=1}^{N-1} J_{t_{k}, t_{k+1}}\left(\Psi_{k}\right)
$$

where $\Psi$ is assumed to be of the form (13).

Lemma 6 The space $\Lambda_{0}^{S}\left(\Pi_{p}(E, F)\right)$ is dense in $\Lambda\left(\Pi_{p}(E, F)\right)$ w.r.t. $\|\cdot\|_{\Lambda}$.

Proof The result follows from the construction in the proof of [6, Prop. 4.22(ii)].

Theorem 7 (Stochastic integration) Assume that the cylindrical Lévy process L has the characteristics $(b, 0, v)$ and satisfies

$$
\int_{E}\left|x^{*}(x)\right|^{p} v(\mathrm{~d} x)<\infty, \quad \text { for all } x^{*} \in E^{*}
$$


and that $F$ is of martingale type $p$ and has the Radon-Nikodym property if $p=1$. Then, the integral operator I defined in (14) is continuous and extends to the operator

$$
I: \Lambda\left(\Pi_{p}(E, F)\right) \rightarrow L^{p}\left(\Omega, \mathcal{F}_{T}, P ; F\right) .
$$

Proof Let $\Psi$ in $\Lambda_{0}^{S}\left(\Pi_{p}(E, F)\right)$ be given by (13) where $\Psi_{k}$ is of the form:

$$
\Psi_{k}=\sum_{i=1}^{m_{k}} 1_{A_{k, i}} \psi_{k, i}
$$

for some disjoint sets $A_{k, 1}, \ldots, A_{k, m_{k}} \in \mathcal{F}_{t_{k}}$ and $\psi_{k, 1}, \ldots, \psi_{k, m_{k}} \in \Pi_{p}(E, F)$ for all $k \in\{0, \ldots, N-1\}$.

The cylindrical Lévy process $L$ can be decomposed into $L(t) x^{*}=B(t) x^{*}+M(t) x^{*}$ for all $x^{*} \in E^{*}$, where $B(t) x^{*}:=t \mathbb{E}\left[L(1) x^{*}\right]$ and $M(t) x^{*}:=L(t) x^{*}-B(t) x^{*}$ for all $x^{*} \in E^{*}$ and $t \geq 0$. Both $B(t): E^{*} \rightarrow L^{1}(\Omega, \mathcal{F}, P ; \mathbb{R})$ and $M(t): E^{*} \rightarrow$ $L^{1}(\Omega, \mathcal{F}, P ; \mathbb{R})$ are linear and continuous since $L(1): E^{*} \rightarrow L^{1}(\Omega, \mathcal{F}, P ; \mathbb{R})$ is continuous due to the closed graph theorem. In particular, both $B$ and $M$ are cylindrical Lévy processes, and we can integrate separately with respect to $B$ and $M$ :

$$
I(\Psi)=I_{B}(\Psi)+I_{M}(\Psi)
$$

For the first integral in (16), we calculate

$$
\left\|I_{B}(\Psi)\right\|^{p}=\sup _{y^{*} \in B_{F^{*}}}\left|y^{*}\left(\int_{0}^{T} \Psi(s) \mathrm{d} B(s)\right)\right|^{p}=\sup _{y^{*} \in B_{F^{*}}}\left|\int_{0}^{T} B(1)\left(\Psi^{*}(s) y^{*}\right) \mathrm{d} s\right|^{p} .
$$

By Hölder's inequality with $q=\frac{p}{p-1}$ and $q=\infty$ if $p=1$, we obtain

$$
\begin{aligned}
\left\|I_{B}(\Psi)\right\|^{p} & \leq \sup _{y^{*} \in B_{F^{*}}} T^{p / q} \int_{0}^{T}\left|B(1)\left(\Psi^{*}(s) y^{*}\right)\right|^{p} \mathrm{~d} s \\
& \leq T^{p / q}\|B(1)\|_{\mathcal{L}\left(E^{*}, \mathbb{R}\right)}^{p} \int_{0}^{T}\left\|\Psi^{*}(s)\right\|_{\mathcal{L}\left(F^{*}, E^{*}\right)}^{p} \mathrm{~d} s .
\end{aligned}
$$

Since $\left\|\Psi^{*}(s)\right\|_{\mathcal{L}\left(F^{*}, E^{*}\right)}=\|\Psi(s)\|_{\mathcal{L}(E, F)} \leq \pi_{p}(\Psi(s))$ according to [9, p. 31], it follows that

$$
\mathbb{E}\left[\left\|I_{B}(\Psi)\right\|^{p}\right] \leq T^{p / q}\|B(1)\|_{\mathcal{L}\left(E^{*}, \mathbb{R}\right)}^{p} \mathbb{E}\left[\int_{0}^{T} \pi_{p}(\Psi(s))^{p} \mathrm{~d} s\right]
$$

For estimating the second term in (16), define the Banach space

$$
R_{p}=\left\{X:(0, T] \times \Omega \rightarrow \mathbb{R}: \text { measurable and } \sup _{t \in(0, T]} \frac{1}{t^{1 / p}}\left(\mathbb{E}\left[|X(t)|^{p}\right]\right)^{1 / p}<\infty\right\}
$$


with the norm $\|X\|_{R_{p}}=\sup _{t \in(0, T]} \frac{1}{t^{1 / p}}\left(\mathbb{E}\left[|X(t)|^{p}\right]\right)^{1 / p}$. By standard properties of the real-valued Lévy martingales, see, for example, [18, Th. 8.23(i)], it follows that there exists a constant $c>0$ such that

$$
\mathbb{E}\left[\left|M(t) x^{*}\right|^{p}\right] \leq c t \int_{\mathbb{R}}|\beta|^{p}\left(v \circ\left(x^{*}\right)^{-1}\right)(\mathrm{d} \beta) \quad \text { for all } x^{*} \in E^{*} .
$$

Here, we use that the Lévy measure of $M(1) x^{*}$ is given by $v \circ\left(x^{*}\right)^{-1}$. It follows that we can consider the map $M: E^{*} \rightarrow R_{p}$ defined by $M x^{*}=\left(M(t) x^{*}: t \in(0, T]\right)$. To show that $M$ is continuous, let $x_{n}^{*}$ converges to $x^{*}$ in $E^{*}$ and $M x_{n}^{*}$ to some $Y$ in $R_{p}$. It follows that $M(t) x_{n}^{*} \rightarrow Y(t)$ in $L^{p}(\Omega ; \mathbb{R})$ for every $t \in(0, T]$. On the other hand, continuity of $M(t): E^{*} \rightarrow L^{1}(\Omega, \mathcal{F}, P ; \mathbb{R})$ implies $M(t) x_{n}^{*} \rightarrow M(t) x^{*}$ in $L^{0}(\Omega ; \mathbb{R})$. Thus, $Y(t)=M(t) x^{*}$ for all $t \in(0, T]$ a.s., and the closed graph theorem satisfies that $M: E^{*} \rightarrow R_{p}$ is continuous. It follows that

$$
\left\|M\left(t_{k+1}-t_{k}\right)\right\|_{L\left(E^{*} ; L^{p}(\Omega ; \mathbb{R})\right)}^{p} \leq\left(t_{k+1}-t_{k}\right)\|M\|_{\mathcal{L}\left(E^{*}, R_{p}\right)}^{p} .
$$

Let $J_{t_{k}, t_{k+1}}$ be the operators defined in (7) with $L$ replaced by $M$. Since $F$ is of martingale type $p$, here exists a constant $C_{p}>0$ such that Lemma 5 and inequality (19) imply

$$
\begin{aligned}
\mathbb{E}\left[\left\|I_{M}(\Psi)\right\|^{p}\right] & =\mathbb{E}\left[\left\|\sum_{k=1}^{N-1} J_{t_{k}, t_{k+1}}\left(\Psi_{k}\right)\right\|^{p}\right] \\
& \leq C_{p} \mathbb{E}\left[\sum_{k=1}^{N-1}\left\|J_{t_{k}, t_{k+1}}\left(\Psi_{k}\right)\right\|^{p}\right] \\
& \leq C_{p} \sum_{k=1}^{N-1}\left\|M\left(t_{k+1}-t_{k}\right)\right\|_{\mathcal{L}\left(E^{*} ; L^{p}(\Omega ; \mathbb{R})\right)}^{p} \mathbb{E}\left[\pi_{p}\left(\Psi_{k}\right)^{p}\right] \\
& \leq C_{p}\|M\|_{\mathcal{L}\left(E^{*}, R_{p}\right)}^{p} \mathbb{E}\left[\int_{0}^{T} \pi_{p}(\Psi(s))^{p} \mathrm{~d} s\right] .
\end{aligned}
$$

Together with (17), this completes the proof.

By rewriting Condition (15) as

$$
\begin{aligned}
& \int_{B_{\mathbb{R}}}|\beta|^{p}\left(\nu \circ\left(x^{*}\right)^{-1}\right)(\mathrm{d} \beta)<\infty \text { and } \\
& \int_{B_{\mathbb{R}}^{c}}|\beta|^{p}\left(\nu \circ\left(x^{*}\right)^{-1}\right)(\mathrm{d} \beta)<\infty \text { for all } x^{*} \in E^{*},
\end{aligned}
$$

it follows that Condition (15) is equivalent to

$\left(L(t) x^{*}: t \geq 0\right)$ is $p$-integrable and has finite $p$-variation for each $x^{*} \in E^{*}$. 
This is a natural requirement if we want to control the moments, see $[2,16]$ and Remark 9. Condition (15) implies in particular that the Blumenthal-Getoor index of $\left(L(t) x^{*}: t \geq 0\right)$ is at most $p$. The interplay between the integrability of the Lévy process and its Blumenthal-Getoor index was observed also in [4,5].

Example 8 (Gaussian case) Note that if $p<2$, then $L$ cannot have the Gaussian part for the assertion to hold. Indeed, let $W$ be a one-dimensional Brownian motion and suppose for contradiction that

$$
\mathbb{E}\left[\left|\int_{0}^{T} \Psi(t) \mathrm{d} W(t)\right|^{p}\right] \leq C \mathbb{E}\left[\int_{0}^{T}|\Psi(t)|^{p} \mathrm{~d} t\right]
$$

for some constant $C$ and every real-valued predictable process $\Psi$ with $\mathbb{E}\left[\int_{0}^{T}|\Psi(t)|^{2} \mathrm{~d} t\right]$ $<\infty$. Choose for each $n \in \mathbb{N}$ the stochastic process $\Psi_{n}(t)=\mathbb{1}_{[0,1 / n]}(t)$ for $t \in[0, T]$. By [10, Sec. 3.478], we calculate

$$
\mathbb{E}\left[\left|\int_{0}^{T} \Psi_{n}(t) \mathrm{d} W(t)\right|^{p}\right]=\mathbb{E}\left[\left|W\left(\frac{1}{n}\right)\right|^{p}\right]=\left(\frac{1}{n}\right)^{\frac{p}{2}} \frac{2^{\frac{p}{2}} \Gamma\left(\frac{p+1}{2}\right)}{\sqrt{\pi}} .
$$

But on the other side, since $\mathbb{E}\left[\int_{0}^{T}\left|\Psi_{n}(t)\right|^{p} \mathrm{~d} t\right]=\frac{1}{n}$, solving (20) for $n$ yields

$$
n^{1-\frac{p}{2}} \leq \frac{C \sqrt{\pi}}{2^{\frac{p}{2}} \Gamma\left(\frac{p+1}{2}\right)}
$$

which results in a contradiction by taking the limit as $n \rightarrow \infty$.

Example 9 (Stable case) Let $E=L^{p^{\prime}}(\mathcal{O})$ for $p^{\prime}=p /(p-1)$ and some $\mathcal{O} \subseteq \mathbb{R}^{d}$. The canonical $\alpha$-stable cylindrical Lévy process has the characteristic function $\phi_{L(1)}\left(x^{*}\right)=\exp \left(-\left\|x^{*}\right\|^{\alpha}\right)$ for each $x^{*} \in E^{*}$; see [24]. It follows that the realvalued Lévy process $\left(L(t) x^{*}: t \geq 0\right)$ is symmetric $\alpha$-stable with Lévy measure $\nu \circ\left(x^{*}\right)^{-1}(\mathrm{~d} \beta)=c \frac{1}{|\beta|^{1+\alpha}} \mathrm{d} \beta$ for a constant $c>0$. Condition (15) fails to hold since

$$
\begin{gathered}
\int_{B_{\mathbb{R}}}|\beta|^{p} v \circ\left(x^{*}\right)^{-1}(\mathrm{~d} \beta)=\infty \text { for } p \leq \alpha, \\
\int_{B_{\mathbb{R}}^{c}}|\beta|^{p} v \circ\left(x^{*}\right)^{-1}(\mathrm{~d} \beta)=\infty \text { for } p \geq \alpha .
\end{gathered}
$$

One can observe in a similar way as in the Gaussian case that the stochastic integral operator with respect to the $\alpha$-stable cylindrical Lévy process $L$ is not continuous. If $\Psi_{n}(t)=\mathbb{1}_{[0,1 / n]}(t)$, then in the inequality

$$
\mathbb{E}\left[\left|\int_{0}^{T} \Psi_{n}(t) \mathrm{d} L(t)\right|^{p}\right] \leq C \mathbb{E}\left[\int_{0}^{T}\left|\Psi_{n}(t)\right|^{p} \mathrm{~d} t\right]
$$


the left-hand side is infinite for $p \geq \alpha$. For $p<\alpha$, we use the self-similarity of the stable processes to calculate

$$
\mathbb{E}\left[\left|\int_{0}^{T} \Psi_{n}(t) \mathrm{d} L(t)\right|^{p}\right]=\mathbb{E}\left[\left|L\left(\frac{1}{n}\right)\right|^{p}\right]=\mathbb{E}\left[\frac{1}{n^{p / \alpha}}|L(1)|^{p}\right] .
$$

Solving (21) for $n$ yields

$$
n^{(\alpha-p) / \alpha} \leq \frac{C}{\mathbb{E}\left[|L(1)|^{p}\right]}
$$

which results in a contradiction by taking the limit as $n \rightarrow \infty$.

Therefore, the stochastic integral mapping with respect to the $\alpha$-stable process cannot be continuous as a mapping from $L^{p}([0, T] \times \Omega, \mathcal{P}, \mathrm{d} t \otimes P ; \mathbb{R})$ to $L^{p}\left(\Omega, \mathcal{F}_{T}, P ; \mathbb{R}\right)$ for any $p>0$. A moment inequality with different powers on the left- and right-hand sides was proven in the case of real-valued integrands and vector-valued integrators in [25]. They prove for any $\alpha$-stable Lévy process $L$ and $p<\alpha$ that

$$
\mathbb{E}\left[\left(\sup _{t \leq T}\left\|\int_{0}^{t} \Psi(s) \mathrm{d} L(s)\right\|\right)^{p}\right] \leq C \mathbb{E}\left[\left(\int_{0}^{T}|\Psi(s)|^{\alpha} \mathrm{d} t\right)^{p / \alpha}\right]
$$

Example 10 In various publications, e.g. [15,19,20,23], specific examples of the following kind of a cylindrical Lévy process have been studied: let $E$ be a Hilbert space with an orthonormal basis $\left(e_{k}\right)$ and let $L$ be given by

$$
L(t) x=\sum_{k=1}^{\infty}\left\langle x, e_{k}\right\rangle \ell_{k}(t) \quad \text { for all } x \in E
$$

where $\left(\ell_{k}\right)$ is a sequence of independent, one-dimensional Lévy processes $\ell_{k}$ with characteristics $\left(b_{k}, 0, \rho_{k}\right)$. Precise conditions under which the sum converges and related results can be found in [23]. In this case, we claim that Condition (15) is satisfied if and only if

$$
\sum_{k=1}^{\infty}\left(\int_{\mathbb{R}}|\beta|^{p} \rho_{k}(\mathrm{~d} \beta)\right)^{\frac{2}{2-p}}<\infty
$$

It is shown in [23, Lem. 4.2] that the cylindrical Lévy measure $v$ of $L$ is given by

$$
v(A)=\sum_{k=1}^{\infty} \rho_{k} \circ m_{e_{k}}^{-1}(A) \quad \text { for all } A \in \mathcal{Z}(E)
$$


where $m_{e_{k}}: \mathbb{R} \rightarrow E$ is given by $m_{e_{k}}(x)=x e_{k}$. Condition (15) simplifies to

$$
\begin{aligned}
\int_{E}|\langle y, x\rangle|^{p} v(\mathrm{~d} x) & =\sum_{k=1}^{\infty} \int_{E}|\langle y, x\rangle|^{p}\left(\rho_{k} \circ m_{e_{k}}^{-1}\right)(\mathrm{d} x) \\
& =\sum_{k=1}^{\infty}\left|\left\langle y, e_{k}\right\rangle\right|^{p} \int_{\mathbb{R}}|\beta|^{p} \rho_{k}(\mathrm{~d} \beta)<\infty
\end{aligned}
$$

for any $y \in E$. This is equivalent to

$$
\sum_{k=1}^{\infty} \alpha_{k} \int_{\mathbb{R}}|\beta|^{p} \rho_{k}(\mathrm{~d} \beta)<\infty \quad \text { for any }\left(\alpha_{k}\right) \in \ell^{2 / p}\left(\mathbb{R}_{+}\right)
$$

which results in $\left(\int_{\mathbb{R}}|\beta|^{p} \rho_{k}(\mathrm{~d} \beta)\right)_{k \in \mathbb{N}} \in \ell^{2 / p}(\mathbb{R})^{*}=\ell^{2 /(2-p)}(\mathbb{R})$.

Example 11 Another example is cylindrical compound Poisson process, see, for example, [1, Ex. 3.5]. These are cylindrical Lévy processes of the form:

$$
L(t) x^{*}=\sum_{k=1}^{N(t)} Y_{k} x^{*} \quad \text { for all } x^{*} \in E^{*}
$$

where $N$ is a real-valued Poisson process with intensity $\lambda$ and $Y_{k}$ are identically distributed, cylindrical random variables, independent of $N$, and say with cylindrical distribution $\rho$. Since the Lévy measure of $\left(L(t) x^{*}: t \geq 0\right)$ is given by $\lambda \rho \circ\left(x^{*}\right)^{-1}$, it follows that Condition (15) is satisfied if and only if

$$
\int_{E}\left|x^{*}(x)\right|^{p} \rho(\mathrm{d} x)<\infty \quad x^{*} \in E^{*}
$$

Remark 12 If $p=2$ and $E$ and $F$ are Hilbert spaces, the space of admissible integrands $\Lambda\left(\Pi_{2}(E, F)\right)$ is given by

$$
\left\{\Psi:[0, T] \times \Omega \rightarrow L_{\mathrm{HS}}(E, F): \Psi \text { is predictable and } \mathbb{E}\left[\int_{0}^{T}\|\Psi(s)\|_{L_{\mathrm{HS}}(E, F)}^{2} \mathrm{~d} s\right]<\infty\right\},
$$

as in the work [22]. This is only suboptimal, as it is known that in this case, the space of admissible integrands can be enlarged to predictable processes satisfying

$$
\mathbb{E}\left[\int_{0}^{T}\left\|\Psi(s) Q^{1 / 2}\right\|_{L_{\mathrm{HS}}(E, F)}^{2} \mathrm{~d} s\right]<\infty
$$

where $Q$ is the covariance operator associated with $L$; see [13]. In this way, the space of integrands depends on the Lévy triplet of the integrator. One can ask whether a similar result is possible in our more general setting for $p<2$ and for Banach spaces by replacing the covariance operator by the quadratic variation. 


\section{Existence and Uniqueness of Solution}

In this section, we apply the developed integration theory to derive the existence of an evolution equation in a Banach space under standard assumptions. For this purpose, we consider

$$
\begin{aligned}
\mathrm{d} X(t) & =(A X(t)+B(X(t))) \mathrm{d} t+G(X(t)) \mathrm{d} L(t), \\
X(0) & =X_{0}
\end{aligned}
$$

where $X_{0}$ is an $\mathcal{F}_{0}$-measurable random variable in a Banach space $F$ and the driving noise $L$ is a cylindrical Lévy process in a Banach space $E$ with finite $p$-th weak moments and finite $p$-variation. The operator $A$ is the generator of a $C_{0}$-semigroup on $F$ and $B: F \rightarrow F$ and $G: F \rightarrow \Pi_{p}(E, F)$ are some functions.

Definition 13 A mild solution of (24) is a predictable process $X$ such that

$$
\sup _{t \in[0, T]} \mathbb{E}\left[\|X(t)\|^{p}\right]<\infty
$$

for some $p \geq 1$, and such that, for all $t \in[0, T]$, we have $P$-a.s.

$$
X(t)=S(t) X_{0}+\int_{0}^{t} S(t-s) B(X(s)) \mathrm{d} s+\int_{0}^{t} S(t-s) G(X(s)) \mathrm{d} L(s) .
$$

We assume Lipschitz and linear growth condition on the coefficients $F$ and $G$ and an integrability assumption on the initial condition.

Assumption 14 For fixed $p \in[1,2]$, we assume:

(A1) there exist a function $b \in L^{1}([0, T] ; \mathbb{R})$ such that for any $x, x_{1}, x_{2} \in F$ and $t \in[0, T]:$

$$
\begin{aligned}
\|S(t) B(x)\| & \leq b(t)(1+\|x\|), \\
\left\|S(t)\left(B\left(x_{1}\right)-B\left(x_{2}\right)\right)\right\| & \leq b(t)\left\|x_{1}-x_{2}\right\| .
\end{aligned}
$$

(A2) there exist a function $g \in L^{p}([0, T] ; \mathbb{R})$ such that for any $x, x_{1}, x_{2} \in F$ and $t \in[0, T]:$

$$
\begin{aligned}
\pi_{p}(S(t) G(x)) & \leq g(t)(1+\|x\|), \\
\pi_{p}\left(S(t)\left(G\left(x_{1}\right)-G\left(x_{2}\right)\right)\right) & \leq g(t)\left\|x_{1}-x_{2}\right\| .
\end{aligned}
$$

(A3) $X_{0} \in L^{p}\left(\Omega, \mathcal{F}_{0}, P ; F\right)$.

Theorem 15 Let $p \in[1,2]$ and suppose that the Banach spaces $E$ and $F$ satisfy that

(a) $E$ is reflexive or has separable dual, and $E^{* *}$ has the approximation property,

(b) $F$ is of martingale type $p$,

(c) if $p=1$, then $F$ has the Radon-Nikodym property. 
If $L$ is a cylindrical Lévy process such that (15) holds with some $p \in[1,2]$, then conditions (A1)-(A3) imply that there exists a unique mild solution of (24).

Proof We define the space

$$
\tilde{\mathcal{H}}_{T}:=\left\{X:[0, T] \times \Omega \rightarrow F \text { is predictable and } \sup _{t \in[0, T]} \mathbb{E}\left[\|X(t)\|^{p}\right]<\infty\right\},
$$

and a family of seminorms for $\beta \geq 0$ :

$$
\|X\|_{T, \beta}:=\left(\sup _{t \in[0, T]} e^{-\beta t} \mathbb{E}\left[\|X(t)\|^{p}\right]\right)^{1 / p} .
$$

Let $\mathcal{H}_{T}$ be the set of equivalence classes of elements $\tilde{\mathcal{H}}_{T}$ relative to $\|\cdot\|_{T, 0}$. Define an operator $K: \mathcal{H}_{T} \rightarrow \mathcal{H}_{T}$ by $K(X):=K_{0}(X)+K_{1}(X)+K_{2}(X)$, where

$$
\begin{aligned}
K_{0}(X)(t) & :=S(t) X_{0}, \\
K_{1}(X)(t) & :=\int_{0}^{t} S(t-s) B(X(s)) \mathrm{d} s, \\
K_{2}(X)(t) & :=\int_{0}^{t} S(t-s) G(X(s)) \mathrm{d} L(s) .
\end{aligned}
$$

The Bochner integral and the stochastic integral above are well defined because $X$ is predictable and for every $t \in[0, T]$ the mappings

$$
[0, t] \times F \ni(s, x) \mapsto S(t-s) B(x), \quad[0, t] \times F \ni(s, h) \mapsto S(t-s) G(x)
$$

are continuous. The appropriate integrability condition follows from (26) and (27).

For applying Banach's fixed point theorem, we first show that $K$ indeed maps to $\mathcal{H}_{T}$. Choose constants $m \geq 1$ and $\omega \in \mathbb{R}$ such that $\|S(t)\| \leq m e^{\omega t}$ for each $t \geq 0$. It follows that

$$
\sup _{t \in[0, T]} \mathbb{E}\left[\left\|S(t) X_{0}\right\|^{p}\right] \leq m e^{|\omega| T} \mathbb{E}\left[\left\|X_{0}\right\|^{p}\right]<\infty
$$

By Assumption (A1) and Hölder's inequality, we obtain with $q=\frac{p}{p-1}$ that

$$
\begin{aligned}
& \sup _{t \in[0, T]} \mathbb{E}\left[\left\|\int_{0}^{t} S(t-s) B(X(s)) \mathrm{d} s\right\|^{p}\right] \\
& \leq \sup _{t \in[0, T]} \mathbb{E}\left[\left(\int_{0}^{t} b(t-s)(1+\|X(s)\|) \mathrm{d} s\right)^{p}\right] \\
& \leq \sup _{t \in[0, T]} \mathbb{E}\left[\left(\int_{0}^{t} b(t-s) d s\right)^{p / q} \int_{0}^{t} b(t-s)(1+\|X(s)\|)^{p} \mathrm{~d} s\right]
\end{aligned}
$$




$$
\begin{aligned}
& \leq\left(\int_{0}^{T} b(s) \mathrm{d} s\right)^{p / q} 2^{p-1}\left(1+\|X\|_{T, 0}\right) \sup _{t \in[0, T]} \int_{0}^{t} b(t-s) \mathrm{d} s \\
& =\left(\int_{0}^{T} b(s) \mathrm{d} s\right)^{1+p / q} 2^{p-1}\left(1+\|X\|_{T, 0}\right) \\
& <\infty
\end{aligned}
$$

Similarly, we conclude from Assumption (A2) and Theorem 7 that there exists a constant $c>0$ such that

$$
\begin{aligned}
& \sup _{t \in[0, T]} \mathbb{E}\left[\left\|\int_{0}^{t} S(t-s) G(X(s)) \mathrm{d} L(s)\right\|^{p}\right] \\
& \leq c \sup _{t \in[0, T]} \mathbb{E}\left[\int_{0}^{t} \pi_{p}(S(t-s) G(X(s)))^{p} \mathrm{~d} s\right] \\
& \leq c \sup _{t \in[0, T]} \mathbb{E}\left[\int_{0}^{t} g(t-s)^{p}(1+\|X(s)\|)^{p} \mathrm{~d} s\right] \\
& \leq c 2^{p-1}\left(1+\|X\|_{T, 0}^{p}\right) \int_{0}^{T} g(s)^{p} \mathrm{~d} s \\
& <\infty
\end{aligned}
$$

Next, we establish that $K$ is stochastically continuous. For this purpose, let $\epsilon>0$. For each $t \geq 0$, we obtain

$$
\begin{aligned}
\mathbb{E} & {\left[\left\|K_{1}(t+\epsilon)-K_{1}(t)\right\|\right] } \\
& =\mathbb{E}\left[\left\|\int_{0}^{t+\epsilon} S(t+\epsilon-s) B(X(s)) \mathrm{d} s-\int_{0}^{t} S(t-s) B(X(s)) \mathrm{d} s\right\|\right] \\
& =\mathbb{E}\left[\left\|\int_{t}^{t+\epsilon} S(t+\epsilon-s) B(X(s)) \mathrm{d} s+\int_{0}^{t}(S(\epsilon)-\mathrm{Id}) S(t-s) B(X(s)) \mathrm{d} s\right\|\right] \\
& \leq \mathbb{E}\left[\int_{t}^{t+\epsilon}\|S(t+\epsilon-s) B(X(s))\| \mathrm{d} s+\int_{0}^{t}\|(S(\epsilon)-\mathrm{Id}) S(t-s) B(X(s))\| \mathrm{d} s\right] \\
& =: I_{1}+I_{2} .
\end{aligned}
$$

Since $\|X(s)\| \leq 1+\|X(s)\|^{p}$ for all $s \geq 0$, it follows, for $\epsilon \rightarrow 0$, that

$$
I_{1} \leq \mathbb{E}\left[\int_{t}^{t+\epsilon} b(t+\epsilon-s)(1+\|X(s)\|) \mathrm{d} s\right] \leq\left(2+\|X(s)\|^{p}\right) \int_{0}^{\epsilon} b(s) \mathrm{d} s \rightarrow 0 .
$$

With the same estimate $\|X(s)\| \leq 1+\|X(s)\|^{p}$, we obtain

$$
\begin{aligned}
\|(S(\epsilon)-\mathrm{Id}) S(t-s) B(X(s))\| & \leq\left(1+m e^{|\omega|}\right) b(t-s)(1+\|X(s)\|) \\
& \leq\left(2+\|X\|_{T, 0}^{p}\right)\left(1+m e^{|\omega|}\right) b(t-s) .
\end{aligned}
$$


Since the integrand of $I_{2}$ tends to 0 as $\epsilon \rightarrow 0$ by the strong continuity of the semigroup, Lebesgue's dominated convergence theorem shows that $I_{2}$ tends to 0 as $\epsilon \rightarrow 0$.

For $K_{2}$, we obtain by Theorem 7 that there exists a constant $c>0$ such that

$$
\begin{aligned}
\mathbb{E}[ & \left.\left\|K_{2}(t+\epsilon)-K_{2}(t)\right\|^{p}\right] \\
= & \mathbb{E}\left[\left\|\int_{0}^{t+\epsilon} S(t+\epsilon-s) G(X(s)) \mathrm{d} L(s)-\int_{0}^{t} S(t-s) G(X(s)) \mathrm{d} L(s)\right\|^{p}\right] \\
= & \mathbb{E}\left[\| \int_{t}^{t+\epsilon} S(t+\epsilon-s) G(X(s)) \mathrm{d} L(s)\right. \\
& \left.+\int_{0}^{t}(S(\epsilon)-\mathrm{Id}) S(t-s) G(X(s)) \mathrm{d} L(s) \|^{p}\right] \\
\leq & 2^{p-1} \mathbb{E}\left[\left\|\int_{t}^{t+\epsilon} S(t+\epsilon-s) G(X(s)) \mathrm{d} L(s)\right\|^{p}\right. \\
& \left.+\left\|\int_{0}^{t}(S(\epsilon)-\mathrm{Id}) S(t-s) G(X(s)) \mathrm{d} L(s)\right\|^{p}\right] \\
\leq & c 2^{p-1} \mathbb{E}\left[\int_{t}^{t+\epsilon} \pi_{p}(S(t+\epsilon-s) G(X(s)))^{p} \mathrm{~d} s\right. \\
& \left.+\int_{0}^{t} \pi_{p}((S(\epsilon)-\mathrm{Id}) S(t-s) G(X(s)))^{p} \mathrm{~d} s\right] \\
\leq & c 2^{p-1} \mathbb{E}\left[\int_{t}^{t+\epsilon} 2^{p-1} g(t+\epsilon-s)^{p}\left(1+\|X(s)\|^{p}\right) \mathrm{d} s\right. \\
& \left.+\int_{0}^{t} \pi_{p}((S(\epsilon)-\mathrm{Id}) S(t-s) G(X(s)))^{p} \mathrm{~d} s\right] \\
= & c 2^{p-1}\left(2^{p-1} J_{1}+J_{2}\right),
\end{aligned}
$$

where

$$
\begin{aligned}
J_{1} & =\mathbb{E}\left[\int_{t}^{t+\epsilon} g(t+\epsilon-s)^{p}\left(1+\|X(s)\|^{p}\right) \mathrm{d} s\right] \\
& \leq\left(1+\|X\|_{T, 0}^{p}\right) \int_{t}^{t+\epsilon} g(t+\epsilon-s)^{p} \mathrm{~d} s \rightarrow 0
\end{aligned}
$$

as $\epsilon \rightarrow 0$, and

$$
J_{2}=\mathbb{E}\left[\int_{0}^{t} \pi_{p}((\mathrm{Id}-S(\epsilon)) S(t-s) G(X(s)))^{p} \mathrm{~d} s\right] .
$$

By Theorem 3, the integrand $\pi_{p}((\operatorname{Id}-S(\epsilon)) S(t-s) G(X(s)))^{p}$ converges to 0 for all $t$ and $\omega \in \Omega$. Moreover, it is bounded by $\left(1+m e^{|\omega|}\right)^{p} g(t-s)^{p}(1+\|X(s)\|)^{p}$, which is $\mathrm{d} t \otimes P$-integrable. Thus, Lebesgue's theorem on dominated convergence implies that $J_{2} \rightarrow 0$ as $\epsilon \rightarrow 0$ which completes the proof of stochastic continuity of $K$. In 
particular, stochastic continuity guarantees the existence of a predicable modification of $K$ by [18, Prop. 3.21]. In summary, we obtain that $K$ maps $\mathcal{H}_{T}$ to $\mathcal{H}_{T}$.

For applying Banach's fixed point theorem, it is enough to show that $K$ is a contraction for some $\beta$. We have

$$
\begin{aligned}
\left\|K\left(X_{1}\right)-K\left(X_{2}\right)\right\|_{T, \beta}^{p} \leq & 2^{p-1}\left(\left\|K_{1}\left(X_{1}\right)-K_{1}\left(X_{2}\right)\right\|_{T, \beta}\right. \\
& \left.+\left\|K_{2}\left(X_{1}\right)-K_{2}\left(X_{2}\right)\right\|_{T, \beta}\right) .
\end{aligned}
$$

For the part corresponding to the drift, we calculate similarly to [18, Th. 9.29]

$$
\begin{aligned}
& \left\|K_{1}\left(X_{1}\right)-K_{1}\left(X_{2}\right)\right\|_{T, \beta}^{p} \\
& \leq \sup _{t \in[0, T]} e^{-\beta t} \mathbb{E}\left[\left(\int_{0}^{t} b(t-s)\left\|X_{1}(s)-X_{2}(s)\right\| \mathrm{d} s\right)^{p}\right] \\
& \left.=\sup _{t \in[0, T]} e^{-\beta t} \mathbb{E}\left[\left(\int_{0}^{t} b(t-s)^{1 / q} b(t-s)^{1 / p} \| X_{1}(s)\right)-X_{2}(s) \| \mathrm{d} s\right)^{p}\right] \\
& \left.\leq\left(\int_{0}^{T} b(t-s) \mathrm{d} s\right)^{p / q} \sup _{t \in[0, T]} e^{-\beta t} \int_{0}^{t} b(t-s) \mathbb{E}\left[\| X_{1}(s)\right)-X_{2}(s) \|^{p}\right] \mathrm{d} s \\
& =\left(\int_{0}^{T} b(s) \mathrm{d} s\right)^{p / q} \sup _{t \in[0, T]} e^{-\beta t} \int_{0}^{t} b(t-s) e^{\beta s} e^{-\beta s} \mathbb{E}\left[\left\|X_{1}(s)-X_{2}(s)\right\|^{p}\right] \mathrm{d} s \\
& \leq\left(\int_{0}^{T} b(s) \mathrm{d} s\right)^{p / q}\left\|X_{1}-X_{2}\right\|_{T, \beta}^{p} \sup _{t \in[0, T]} \int_{0}^{t} b(t-s) e^{-\beta(t-s)} \mathrm{d} s \\
& =C(\beta)\left\|X_{1}-X_{2}\right\|_{T, \beta}^{p}
\end{aligned}
$$

with $C(\beta)=\left(\int_{0}^{T} b(s) \mathrm{d} s\right)^{p / q} \int_{0}^{T} b(s) e^{-\beta s} \mathrm{~d} s \rightarrow 0$ as $\beta \rightarrow \infty$.

In the following calculation for the part corresponding to the diffusion, we use in the first inequality the continuity of the stochastic integral formulated in Theorem 7 :

$$
\begin{aligned}
& \left\|K_{2}\left(X_{1}\right)-K_{2}\left(X_{2}\right)\right\|_{T, \beta}^{p} \\
& \leq c \sup _{t \in[0, T]} e^{-\beta t} \mathbb{E}\left[\int_{0}^{t} \pi_{p}\left(S(t-s)\left(G\left(X_{1}(s)\right)-G\left(X_{2}(s)\right)\right)\right)^{p} \mathrm{~d} s\right] \\
& \leq c \sup _{t \in[0, T]} e^{-\beta t} \mathbb{E}\left[\int_{0}^{t} g(t-s)^{p}\left\|X_{1}(s)-X_{2}(s)\right\|^{p} \mathrm{~d} s\right] \\
& =c \sup _{t \in[0, T]} e^{-\beta t} \mathbb{E}\left[\int_{0}^{t} g(t-s)^{p} e^{\beta s} e^{-\beta s}\left\|X_{1}(s)-X_{2}(s)\right\|^{p} \mathrm{~d} s\right] \\
& \leq c\left\|X_{1}-X_{2}\right\|_{T, \beta}^{p} \sup _{t \in[0, T]} \int_{0}^{t} e^{-\beta(t-s)} g(t-s)^{p} \mathrm{~d} s \\
& =C^{\prime}(\beta)\left\|X_{1}-X_{2}\right\|_{T, \beta}^{p},
\end{aligned}
$$


where $C^{\prime}(\beta)=c \int_{0}^{T} e^{-\beta s} g(s)^{p} \mathrm{~d} s \rightarrow 0$ as $\beta \rightarrow \infty$. Consequently, Banach's fixed point theorem implies that there exists a unique $X \in \mathcal{H}_{T}$ such that $K(X)=X$ which completes the proof.

Remark 16 Note that if $E$ and $F$ are Hilbert spaces, then they satisfy assumption (ii) in Theorem 7, see, for example, [27, Cor. 1, p. 109]. Thus for $p=2$, we recover [22].

Remark 17 For processes of the form (22), the integrability assumption can be relaxed to include, for example, stable processes in the same way as in [14] where the existence of variational solutions is demonstrated. The details can be found in the $\mathrm{PhD}$ thesis of the first author.

Acknowledgements I would like to thank Przemysław Wojtaszczyk for a helpful discussion and Emilio Fedele for suggesting to use the finite rank operators in the proof of Theorem 3.

Open Access This article is licensed under a Creative Commons Attribution 4.0 International License, which permits use, sharing, adaptation, distribution and reproduction in any medium or format, as long as you give appropriate credit to the original author(s) and the source, provide a link to the Creative Commons licence, and indicate if changes were made. The images or other third party material in this article are included in the article's Creative Commons licence, unless indicated otherwise in a credit line to the material. If material is not included in the article's Creative Commons licence and your intended use is not permitted by statutory regulation or exceeds the permitted use, you will need to obtain permission directly from the copyright holder. To view a copy of this licence, visit http://creativecommons.org/licenses/by/4.0/.

\section{References}

1. Applebaum, D., Riedle, M.: Cylindrical Lévy processes in Banach spaces. Proc. Lond. Math. Soc. 101(3), 697-726 (2010)

2. Bié, E.S.L.: Étude d'une edps conduite par un bruit poissonnien. Prob. Theory Related Fields 111(2), 287-321 (1998)

3. Brzeźniak, Z., Long, H.: A note on $\gamma$-radonifying and summing operators. In: Chojnowska-Michalik, A., Peszat, S., Stettner, L. (eds.) Stochastic Analysis, Banach Center Publ., vol. 105, pp. 43-57. Polish Acad. Sci. Inst. Math., Warsaw (2015)

4. Chong, C.: Lévy-driven Volterra equations in space and time. J. Theoret. Probab. 30(3), 1014-1058 (2017)

5. Chong, C.: Stochastic PDEs with heavy-tailed noise. Stochastic Process. Appl. 127(7), 2262-2280 (2017)

6. Da Prato, G., Zabczyk, J.: Stochastic Equations in Infinite Dimensions. Cambridge University Press, Cambridge (2014)

7. Dettweiler, E.: Banach space valued processes with independent increments and stochastic integration. In: Dold, A., Eckmann, B. (eds.) Probability in Banach Spaces, IV (Oberwolfach, 1982), Lecture Notes in Math., vol. 990, pp. 54-83. Springer, Berlin (1983)

8. Dettweiler, E.: Stochastic integration of Banach space valued functions. In: Arnold, L., Kotelenez, P. (eds.) Stochastic Space-Time Models and Limit Theorems, pp. 53-59. D. Reidel Publishing Company, Dordrecht (1985)

9. Diestel, J., Jarchow, H., Tonge, A.: Absolutely Summing Operators. Cambridge University Press, Cambridge (1995)

10. Gradshteyn, I.S., Ryzhik, I.M.: Table of Integrals, Series, and Products. Academic Press, Cambridge (2007)

11. Hytönen, T., van Neerven, J., Veraar, M., Weis, L.: Analysis in Banach Spaces. Martingales and Littlewood-Paley Theory, vol. I. Springer, Cham (2016)

12. Jakubowski, A., Riedle, M.: Stochastic integration with respect to cylindrical lévy processes. Ann. Probab. 45(6B), 4273-4306 (2017) 
13. Kosmala, T.: Stochastic partial differential equations driven by cylindrical Lévy processes. Ph.D. thesis King's College London (2019) (submitted)

14. Kosmala, T., Riedle, M.: Variational solutions of stochastic partial differential equations with cylindrical Lévy noise. arXiv:1807.11418

15. Liu, Y., Zhai, J.: A note on time regularity of generalized Ornstein-Uhlenbeck processes with cylindrical stable noise. C. R. Math. Acad. Sci. Paris 350(1-2), 97-100 (2012)

16. Luschgy, H., Pagès, G.: Moment estimates for Lévy processes. Electron. Commun. Probab. 13, 422-434 (2008)

17. Mandrekar, V., Rüdiger, B.: Stochastic Integration in Banach Spaces. Theory and Applications. Springer, Cham (2015)

18. Peszat, S., Zabczyk, J.: Stochastic Partial Differential Equations with Lévy Noise. An Evolution Equation Approach. Cambridge University Press, Cambridge (2007)

19. Peszat, S., Zabczyk, J.: Time regularity of solutions to linear equations with Lévy noise in infinite dimensions. Stochastic Process. Appl. 123(3), 719-751 (2013)

20. Priola, E., Zabczyk, J.: On linear evolution equations for a class of cylindrical Lévy noises. In: Da Prato, G., Tubaro, L. (eds.) Stochastic Partial Differential Equations and Applications, Quad. Mat., vol. 25, pp. 223-242. Dept. Math. Seconda Univ. Napoli, Caserta (2010)

21. Riedle, M.: Infinitely divisible cylindrical measures on Banach spaces. Studia Math. 207(3), 235-256 (2011)

22. Riedle, M.: Stochastic integration with respect to cylindrical Lévy processes in Hilbert spaces: an $L^{2}$ approach. Infin. Dimens. Anal. Quantum Probab. Relat. Top. 17(1), 19 (2014)

23. Riedle, M.: Ornstein-Uhlenbeck processes driven by cylindrical Lévy processes. Potential Anal. 42(4), 809-838 (2015)

24. Riedle, M.: Stable cylindrical Lévy processes and the stochastic Cauchy problem. Electron. Commun. Probab. 23(36), 12 (2018)

25. Rosiński, J., Woyczyński, W.A.: Moment inequalities for real and vector $p$-stable stochastic integrals. In: Probability in Banach Spaces, Proceedings of the International Conference held in Medford, USA, July 16-27, 1984. Springer, Berlin (1985)

26. Saphar, P.: Hypothèse d'approximation à l'ordre $p$ dans les espaces de Banach et approximation d'applications $p$ absolument sommantes. Israel J. Math. 13, 379-399 (1972)

27. Schaefer, H.H., Wolff, M.P.: Topological Vector Spaces. Springer, New York (1999)

28. Schwartz, L.: Applications $p$-sommantes et $p$-radonifiantes. Séminaire d'analyse fonctionnelle (dit “Maurey-Schwartz”), 1972-73. Talk no 3

29. Schwartz, L.: Cylindrical probabilities and $p$-summing and $p$-radonifying maps. In: Seminar Schwartz, Notes on Pure Mathematics 7 (1973)

30. Schwartz, L.: Geometry and Probability in Banach Spaces. Springer, Berlin (1981)

31. Vakhania, N.N., Tarieladze, V.I., Chobanyan, S.A.: Probability Distributions on Banach Spaces. D. Reidel Publishing Co., Dordrecht (1987)

32. van Neerven, J.M.A.M., Veraar, M.C., Weis, L.: Stochastic integration in UMD Banach spaces. Ann. Probab. 35(4), 1438-1478 (2007)

33. Veraar, M., Yaroslavtsev, I.: Cylindrical continuous martingales and stochastic integration in infinite dimensions. Electron. J. Probab. 21(59), 53 (2016)

34. Zhu, J., Brzeźniak, Z., Hausenblas, E.: Maximal inequalities for stochastic convolutions driven by compensated Poisson random measures in Banach spaces. Ann. Inst. Henri Poincaré Probab. Stat. 53(2), 937-956 (2017)

Publisher's Note Springer Nature remains neutral with regard to jurisdictional claims in published maps and institutional affiliations. 